\title{
CONTRIBUTIONS OF APHASIOLOGY TO LANGUAGE EVOLUTION RESEARCH
}

\author{
ANDREAS KYRIACOU \\ Department of Neuropsychology, Zürich University, Binzmühlestrasse 14/1 \\ 8050 Zürich, Country \\ SVERKER JOHANSSON \\ School of Education and Communication, Jönköping University, Box 1026, 55111 \\ Jönköping, Sweden
}

Brain imaging techniques have greatly improved our understanding of language processing in the brain. Nevertheless, finding the neural correlates of linguistic operations seems to be no lesser a quest than in the field of consciousness, for which the mapping between psychological experiences and neural processing has been suggested to be the hard problem (Chalmers, 1995).

Poeppel and Embick (2005) pointed out that psycholinguistics and the neurosciences face a granularity mismatch and an ontological incommensurability problem. In short, they highlight the lack of a common basis of the two disciplines which would allow the formulation of theoretically motivated, biologically grounded and computationally explicit descriptions of language processes in the brain. The dissimilarity concerns both the granularity levels, at which processes are investigated in the two disciplines as well as the respective referenced fundamental elements used. To better align the two disciplines Poeppel and Embick suggest the use of computational models, whose operations must be plausibly executable by neural assemblies and represent subroutines of linguistic computation.

We appreciate Poeppel and Embick's analysis and support their proposal to identify computationally explicit processes. Resorting to computer linguistics is however no guarantee in itself that biologically plausible implementations are identified. Artificial neural networks can be built in a way that mimic human behaviour without being based on a biology-inspired architecture, as exempli- 
fied by the Rumelhart \& McClelland (1986; McClelland \& Patterson, 2002) model of English past tense acquisition.

In order to aid the identification of the relevant atomic processes involved in language perception and production we evaluate a model of language evolution with findings of clinical linguistics, more specifically from studies on agrammatism. Johansson (2005) suggested to work backwards from current grammars through a sequence of possible proto-grammars. The steps between adjacent proto-grammars, in the form of the appearance of new structural elements in language, may indicate likely atomic operations in language processing, added one after the other during the course of language evolution.

Johansson's proposal consists of a four-step hierarchy, which is intended to reflect the appearance of fundamental properties of human language for the existence of which there is wide agreement across different grammar theories: the emergence of structural constraints, most notably with respect to word order; the emergence of hierarchies, i.e. the occurrence of structured units within largerscale structures; the emergence of flexibility in the transformational sense, allowing structures to be moved around; and lastly, the occurrence of recursion, which has been proposed to be the only domain-specific computational capacity involved in language processing (Hauser, Chomsky \& Fitch, 2002, but see also e.g. Pinker \& Jackendoff (2005) and Kinsella (2009)).

We hypothesise that the biologically oldest computing capacities recruited for language processing should be least likely to suffer impairments from brain injuries, and when they do occur, it would be unlikely that they affect language in isolation as these capacities probably evolved pre-linguistically and only later served the processing of language. More recent abilities, such as the proposed capacity for recursion, are more likely to have been selected specifically for linguistic purposes. In contrast to their biologically older counterparts, these capacities may be more vulnerable to break-down and affect specific aspects of language production or perception in isolation.

We use findings from studies investigating the patterns of grammar deficits in aphasia patients to assess the proposed hypothesis and to demonstrate how far aphasiology can contribute to the quest of defining linguistic capacities that are evolutionarily layered and real in both linguistic-computational and neurological terms. We further propose that this methodology can be of more general use in constraining theories of language evolution and proposed intermediate protolanguage stages. 


\section{References}

Chalmers, D.J. (2005) Facing Up to the Problem of Consciousness, Journal of Consciousness Studies, 2, 200-19.

Hauser, M., Chomsky, N. \& Fitch, T. (2002) The Faculty of Language: What Is It, Who Has It, and How Did It Evolve?, Science, 298, 1569-79.

Johansson, S. (2005) Origins of Language: Constraints on Hypotheses. John Benjamins.

Kinsella, A. R. (2009) Language evolution and syntactic theory Cambridge: Cambridge University Press

Pinker, S \& Jackendoff, R (2005) The faculty of language: what's special about it? Cognition 95:201-236

Poeppel, D. \& Embick, D. (2005). The relation between linguistics and neuroscience. In A. Cutler (Ed.), Twenty-First Century Psycholinguistics: Four Cornerstones. Lawrence Erlbaum. 